\title{
Implementation of a structured practical activity to analyse student healthcare worker perceptions and compliance with prescribed infection control procedures
}

Elise S. Pelzer ${ }^{1,2^{*}}$, Zachary Stewart ${ }^{1}$, Holly Peters ${ }^{1}$, Jessica O'Callaghan ${ }^{1}$, Emily Bryan ${ }^{1}$, Lucas Wager ${ }^{1}$ and Juliana Chiruta'

\begin{abstract}
Background: Non-compliance with infection control guidelines has been reported within healthcare settings. Infection control education in undergraduate healthcare education programs forms a critical component in preparing student healthcare workers for vocational roles.

Methods: Clinical sciences students (nutrition science, paramedicine, pharmacy, podiatry, optometry studying for qualifications recognised by the Australian Health Practitioner Regulation Agency) self-reported hygiene perceptions and practices and collected microbiological swabs from personal or medical equipment items before and after recommended disinfection procedures.
\end{abstract}

Results: Cultivable microorganisms were isolated from $95 \%$ of student medical equipment items. Disinfection significantly reduced microbial growth on student medical equipment items $(P<0.05)$.

Conclusions: Student perceptions of infection control procedures do not always correlate with infection control practice. Infection control education of undergraduate healthcare students requires ongoing assessment to ensure successful translation into clinical practice.

Keywords: Healthcare worker, Undergraduate students, Infection control

\section{Background}

Breaking the chain of infection underpins infection prevention and control policy, however, teaching this content to undergraduate students studying health courses is challenging, with infection control frequently seen as boring. Concerningly, healthcare-associated infections (HAIs) are the most common preventable condition affecting hospitalized patients [1]. Each year

\footnotetext{
*Correspondence: e.pelzer@qut.edu.au

${ }^{2}$ Queensland University of Technology, PO Box 2434, Brisbane,

Queensland 4001, Australia

Full list of author information is available at the end of the article
}

in Australia, some 180,000 patients suffer HAIs that increase patient morbidity and mortality risks, resulting in prolonged hospitalization, reduced quality of life, and increased healthcare costs for both patients and the healthcare system [1]. Frequent-touch personal and medical equipment used by healthcare workers in performing their duties is a well-accepted vector for the transmission of microorganisms including drug resistant pathogens, which are capable of surviving on fomites for a period of hours to months, but are invisible to the naked eye [7]. Research indicates that contamination of clothing and small medical equipment items including stethoscopes and pen lights is 
substantial, and is equivalent to that observed on the hands of healthcare workers $(\mathrm{HCW})$ [9]. Knowledge of the ability of clothing and medical items to act as vectors for carriage of transient microorganisms is critical for reinforcing $\mathrm{HCW}$ understanding of procedures that can interrupt the chain of infection [12].

Infection prevention and control practices aim to control and reduce the risk of HAI transmission by breaking the chain of infection between the microbe and the susceptible host: HCW, fomites, and patients. Regulatory bodies, including The Centers for Disease Control and Prevention (CDC) and The National Health and Medical Research Council (NHMRC), provide infection control guidelines for healthcare workers [11]. Allied health boards subscribe to these same infection control guidelines, supporting the need for inclusion of opportunities to embed these guidelines in undergraduate clinical sciences teaching programs (Australian Health Practitioner Regulation Agency). However, evidence suggests that HCWs demonstrate poor compliance with infection control protocols, potentially as a result of limited infectious disease knowledge [2, 3].

Understanding and implementing effective and safe infection control begins at the training level when student healthcare workers undertake their vocational training at educational institutions $[13,14]$. Previous studies report a suboptimal commitment to cleaning and disinfection of personal and medical equipment items by both clinical sciences students and experienced HCWs [6]. The aim of this study was to assess the microbial load on undergraduate clinical sciences (nutrition science, paramedicine, pharmacy, podiatry, optometry studying for qualifications recognised by the Australian Health Practitioner Regulation Agency) student's personal or medical equipment items to: (1) create a tangible inquiry based learning opportunity regarding the presence of "invisible" microorganisms and infectious disease transmission routes; (2) determine whether student hygiene perceptions and practice matched those prescribed by NHMRC prevention and control of infectious disease guidelines; and (3) leverage the results to highlight the efficacy of a simple disinfection procedure on reducing the risk of infectious disease transmission.

\section{Methods}

\section{Participants}

Undergraduate students enrolled in second- or third-year clinical science (nutrition science, paramedicine, pharmacy, podiatry, optometry studying for qualifications recognised by the Australian Health Practitioner Regulation Agency) units undertaking a mandatory single microbiology focused infection control unit.

\section{Data collection and analysis}

Data collection for this pilot study employed convenience sampling, whereby all students enrolled in the mandatory infection control units were invited to participate in the research project. Students were required to complete a survey specifically developed for this study that was administered prior to laboratory work. The survey settings used a combination of short response questions and questions assessed using a 5-point Likert scale; these questions were designed to obtain data relating to students' self-reported infection control practice (frequency of compliance with prescribed infection control guidelines) and perceived importance of infection control practices. Students were asked to rate their answers from 1 (strongly disagree) to 5 (strongly agree).

Data analysis involved relating student self-reported infection control practices and disinfection procedure/s for personal or medical equipment with the microbiological test culture results obtained from sampling the item during the laboratory classes. Personal items included clothing and personal protective equipment described in the literature as a transmission source of transient microorganisms and medical equipment included items used to perform medical assessments on patients where the student had already participated in clinical placement rotations. As part of the survey, students created a unique code for their data set to de-identify the data. Student creation of the code ensured that the research staff remained blinded to the identity of all student samples. The codes were used by research staff for the purpose of matching survey data and samples for data analysis.

\section{Microbiology screening}

Students were encouraged to select relevant personal or medical equipment items independently due to constraints surrounding the diverse student cohort, discipline-specific timing of clinical placement, and required medical items. This process ensured inquiry-based student learning opportunities to meet the objective of demonstrating to students that simple and regular disinfection of items (both personal and medical) reduces the risk of transmission of potential pathogens to themselves and their patients. Specimens for microbiological screening of students' personal or medical equipment items were collected by students aseptically using sterile swabs moistened in sterile distilled water (Edwards, Narellan, New South Wales). Specimens were inoculated onto Columbia Horse Blood Agar Plates (Edwards) and incubated for $18-24 \mathrm{~h}$ at $37^{\circ} \mathrm{C}$ in air. All inoculated plates were examined for microbial growth, the number of colony-forming units (CFU) where growth was present, and colonial morphology were recorded. 


\section{Statistical analysis}

All statistical tests were performed using $\mathrm{R} 3.4 .2$ software [15]. Microbial counts were categorised ordinally to enable statistical tests to be performed, as too numerous to count (TNTC) observations were not capable of being converted into continuous values. Categories correspond to whole integers on a $\log 10$ scale; highest to lowest of ordinal scale $=$ TNTC $>101-$ $340 \mathrm{CFU}>11-100 \mathrm{CFU}>1-10 \mathrm{CFU}>0 \mathrm{CFU}$ (no growth). Microbial diversity was measured as the number of morphologically distinctive colony types visually evident on cultured media.

Wilcoxon signed rank test, with continuity correction, comparing paired results before and after treatment was performed to ascertain whether the in-class treatment was effective for reducing the number of viable microbes on each student's medical item. The proportion of items with reduced growth after treatment, i.e. colony counts, were categorised into a lower growth category after treatment was calculated.

Additional Wilcoxon signed rank test with continuity correction analysis was performed with paired results, before and after treatment, to determine whether in-class treatment reduced the diversity of cultivable microbes on each student's medical item.

Additional analysis was completed to determine whether the medical items of students who provided certain answers to the quiz questions were more likely to have higher cultured microbial counts or diversity than others. Kruskal-Wallis rank sum tests were used to test this hypothesis, with subsequent utilisation of Dunn's test of multiple comparisons to further investigate any significant results, to identify which quiz responses were correlated to microbial growth or diversity results.

\section{Results}

A total of 93 student data sets (survey responses combined with microbiology culture data) were available for analysis. Identifiable datasets were excluded from analysis.

\section{Microbiological investigations}

Medical and personal items screened by undergraduate clinical sciences students included: stethoscopes (36.6\%), pens $(15.1 \%$, which included responses such as "marker pen", "biro", "ink pen", and "writing pen"), mobile phones $(11.8 \%)$, pen lights $(7.5 \%)$, safety glasses $(7.5 \%)$, glasses (5.4\%, corrective eyewear), clothes (4.3\%), scissors (3.2\% scissors and trauma sheers), and other (8.6\%, such as laptop) (Table 1).

Cultivable microorganisms were isolated from 94.7\% of personal or medical equipment items prior to in-class
Table 1 Students' personal or medical equipment items

\begin{tabular}{lll}
\hline Medical equipment item & Number of items & $\%$ \\
\hline Stethoscope & 34 & 36.6 \\
Pen & 14 & 15.1 \\
Mobile phone & 11 & 11.8 \\
Other & 8 & 8.6 \\
Pen light & 7 & 7.5 \\
Safety glasses & 7 & 7.5 \\
Glasses & 5 & 5.4 \\
Clothes & 4 & 4.3 \\
Scissors & 3 & 3.2 \\
Total & 93 & 100.0 \\
\hline
\end{tabular}

disinfection and from $46.2 \%$ of medical equipment items after in-class disinfection (Table 2). In-class disinfection significantly reduced the number of CFUs cultured from medical equipment items for $80.7 \%$ of medical items $(P<0.0001)$.

Viable counts in the ranges of TNTC, 101-340 CFU and 11-100 CFU, from swabs of personal or medical equipment items were significantly decreased after disinfection when compared to before disinfection ( $P=0.0002, P=0.03$ and $P<0.0001$ respectively). Increased colony counts were observed for the majority of culture plates with initial viable counts of 1-10 CFU or no growth recorded prior to disinfection, indicating that the items were re-contaminated (Table 2, Fig. 1).

Moreover, results from the same test showed that inclass treatment significantly reduced the diversity of CFU cultured on agar after treatment, of which $73.1 \%$ of medical items evinced reduced cultured diversity (morphotypes), when compared to the cultured diversity obtained before treatment $(P<0.0001$, data not shown).

\section{Survey data}

The majority of all respondents agreed or strongly agreed that infection control was critical for protecting self and patients or clients from infectious disease (85.0\%), although more students strongly agreed that it was critical for patients or clients $(69.9 \%)$ rather than for protection of self (58.1\%) (Table 3). Medical item disinfection frequency was low, with the majority of students (53.8\%) admitting that they never disinfected their chosen medical or personal item. Only four students (4.3\% of total) followed proper clinical practice of disinfecting their item after each patient. For students who did elect to clean their item at any frequency, most respondents (30.1\%) used a $70 \%(\mathrm{v} / \mathrm{v})$ ethanol or isopropanol cleaning agent rather than water only $(5.4 \%)$, soap and water $(5.4 \%)$, or some "other" disinfectant-grade cleaning agent (8.6\%). 
Table 2 Microbial load (CFU) on medical equipment item

\begin{tabular}{|c|c|c|c|c|c|c|c|c|c|c|}
\hline \multirow{2}{*}{$\begin{array}{l}\text { Medical equipment item } \\
\text { (number of items) }\end{array}$} & \multicolumn{2}{|l|}{ TNTC } & \multicolumn{2}{|c|}{$101-340$} & \multicolumn{2}{|l|}{$11-100$} & \multicolumn{2}{|l|}{$1-10$} & \multicolumn{2}{|c|}{ No growth } \\
\hline & Before & After & Before & After & Before & After & Before & After & Before & After \\
\hline Clothes (4) & 2 & 1 & 0 & 0 & 1 & 1 & 1 & 0 & 0 & 2 \\
\hline Mobile phone (11) & 1 & 0 & 2 & 1 & 6 & 1 & 1 & 3 & 1 & 6 \\
\hline Pen (14) & 0 & 0 & 0 & 0 & 9 & 0 & 4 & 6 & 1 & 8 \\
\hline Stethoscope (34) & 5 & 0 & 2 & 0 & 14 & 2 & 13 & 13 & 0 & 19 \\
\hline Scissors (3) & 0 & 0 & 1 & 0 & 0 & 0 & 1 & 2 & 1 & 1 \\
\hline Safety glasses (7) & 4 & 0 & 0 & 0 & 2 & 0 & 0 & 5 & 1 & 2 \\
\hline Glasses (5) & 3 & 0 & 1 & 0 & 0 & 0 & 1 & 2 & 0 & 3 \\
\hline Pen light (7) & 0 & 0 & 0 & 0 & 2 & 0 & 4 & 2 & 1 & 5 \\
\hline Other (8) & 1 & 0 & 1 & 0 & 3 & 1 & 3 & 3 & 0 & 4 \\
\hline Total (93) & 16 & 1 & 7 & 1 & 37 & 5 & 28 & 36 & 5 & 50 \\
\hline$\%$ & 17.2 & $1.1^{* *}$ & 7.5 & $1.1^{*}$ & 39.8 & $5.4^{* * *}$ & 30.1 & 38.7 & 5.4 & 53.8 \\
\hline
\end{tabular}

CFU colony forming units, TNTC too numerous to count

${ }^{*} P<0.05,{ }^{* *} P<0.001,{ }^{* * *} P<0.0001$

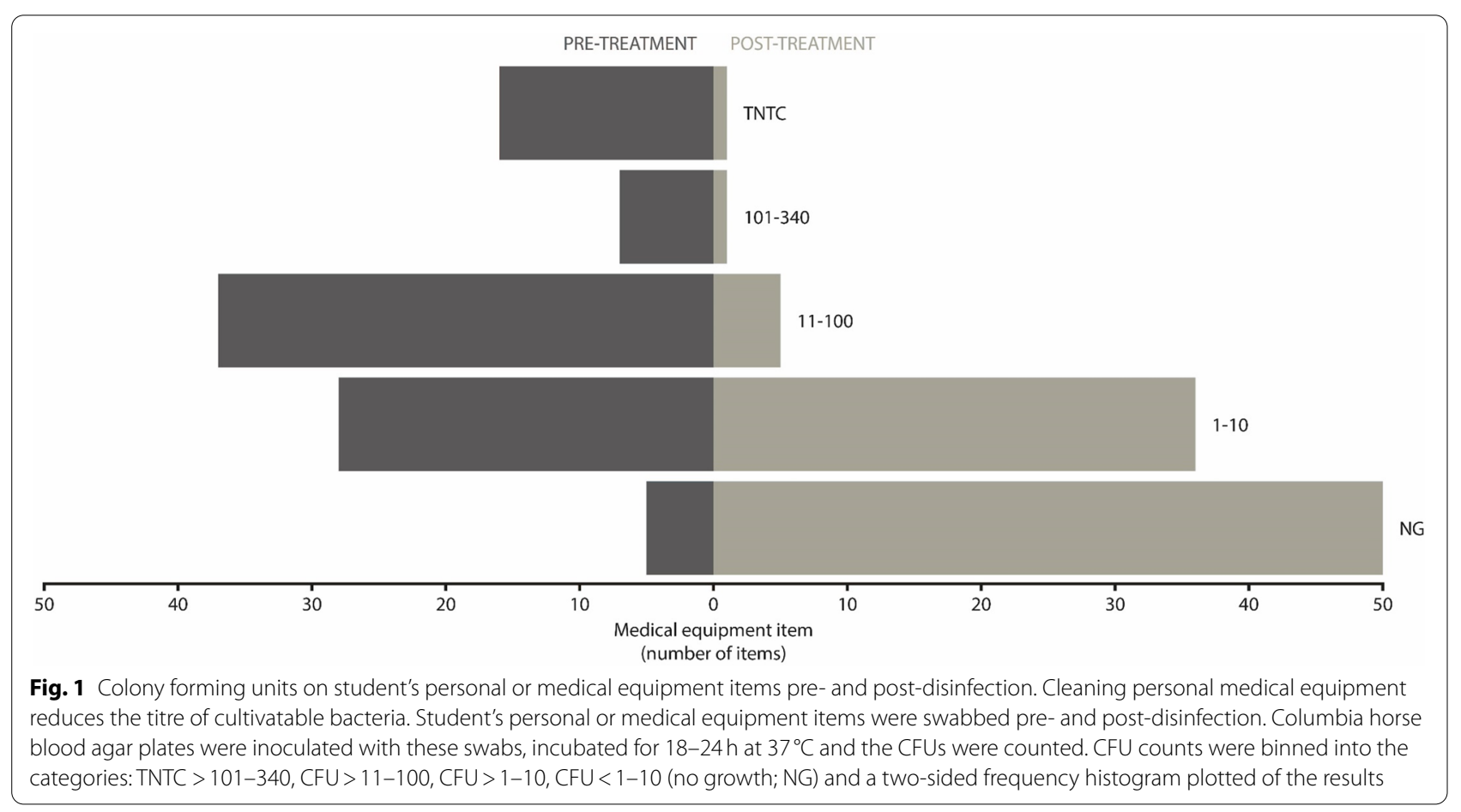

Students were slightly more likely to clean medical items in direct contact with patients $(43.0 \%)$ or themselves $(41.9 \%)$ and less frequently for items in indirect contact with patients (57.0\%).

\section{Correlation between routine disinfection practice and microbial load}

Whilst most items screened would be considered frequent-touch items, certain personal and medical items were more and others less likely to result in the recovery of an increased number of CFUs $(P=0.0323)$. The number of viable CFUs was independent of student perceptions and infection control practices (Supplementary Table 1). Microbial diversity was not significantly correlated with any quiz question response (Supplementary Table 2). Medical equipment items comprised predominantly of non-porous materials, including metals and plastics (pen, pen light, and scissors), could be considered the cleanest items, while glasses (safety glasses and corrective eyewear), clothes, 
Table 3 Infection control perceptions and practices of student HCWs

\begin{tabular}{|c|c|c|}
\hline Variable & $\mathrm{n}=$ & $\%$ \\
\hline \multicolumn{3}{|c|}{ Frequency of medical item disinfection } \\
\hline Never & 50 & $53.8 \%$ \\
\hline Once a year & 10 & $10.8 \%$ \\
\hline Once a week & 12 & $12.9 \%$ \\
\hline Daily & 6 & $6.5 \%$ \\
\hline After each patient & 4 & $4.3 \%$ \\
\hline Other & 11 & $11.8 \%$ \\
\hline \multicolumn{3}{|c|}{ Agents used for cleaning medical equipment } \\
\hline No response & 47 & $50.5 \%$ \\
\hline Water only & 5 & $5.4 \%$ \\
\hline Soap and water & 5 & $5.4 \%$ \\
\hline 70\% ethanol/isopropanol & 28 & $30.1 \%$ \\
\hline Other & 8 & $8.6 \%$ \\
\hline \multicolumn{3}{|c|}{ Frequency of medical equipment item disinfection of components in direct contact with patients } \\
\hline Never & 40 & $43.0 \%$ \\
\hline Once a year & 6 & $6.5 \%$ \\
\hline Once a week & 11 & $11.8 \%$ \\
\hline Daily & 9 & $9.7 \%$ \\
\hline After each patient & 20 & $21.5 \%$ \\
\hline Other & 7 & $7.5 \%$ \\
\hline \multicolumn{3}{|c|}{ Frequency of medical equipment item disinfection of components in indirect contact with patients } \\
\hline Never & 53 & $57.0 \%$ \\
\hline Once a year & 5 & $5.4 \%$ \\
\hline Once a week & 19 & $20.4 \%$ \\
\hline Daily & 7 & $7.5 \%$ \\
\hline After each patient & 3 & $3.2 \%$ \\
\hline Other & 6 & $6.5 \%$ \\
\hline \multicolumn{3}{|c|}{ Frequency of medical equipment item disinfection of components in direct contact with myself } \\
\hline Never & 39 & $41.9 \%$ \\
\hline Once a year & 5 & $5.4 \%$ \\
\hline Once a week & 19 & $20.4 \%$ \\
\hline Daily & 10 & $10.8 \%$ \\
\hline After each patient & 11 & $11.8 \%$ \\
\hline Other & 9 & $9.7 \%$ \\
\hline \multicolumn{3}{|c|}{ Infection control practice is critical to protecting me from infectious diseases } \\
\hline Strongly disagree & 13 & $14.0 \%$ \\
\hline Neutral & 1 & $1.1 \%$ \\
\hline Agree & 25 & $26.9 \%$ \\
\hline Strongly agree & 54 & $58.1 \%$ \\
\hline \multicolumn{3}{|c|}{ Infection control practice is critical to protecting my patients/clients from infectious diseases } \\
\hline Strongly disagree & 14 & $15.1 \%$ \\
\hline Agree & 14 & $15.1 \%$ \\
\hline Strongly agree & 65 & $69.9 \%$ \\
\hline
\end{tabular}


and mobile phones could be considered the dirtiest items (Supplementary Table 1).

\section{Discussion}

Infection prevention and control training in undergraduate HCW courses frequently lacks structured microbiology-based practical activities targeting the chain of infection. HCW student personal and medical equipment items harboured a diverse range of microorganisms, with colony counts often exceeding the normalisation level for clean, classified as less than 20 CFU [12]. Personal and medical equipment items are reported to be potential sources of microbial transmission between HCWs and patients. This project created a unique opportunity to understand the current perceptions and practises of healthcare students and to improve student understanding of the role of infection control practices for safe treatment of patients by providing an opportunity for students to visualise "invisible" microorganisms harboured on items used during patient interactions before and after low-level disinfection. In agreement with previous studies, we confirmed that disinfection significantly reduced the microbial load on personal and medical equipment items. In line with previous reports, alcohol wipes provided an effective method for reducing the microbial load and diversity of microorganisms cultivated from medical and personal equipment items $[8,10,16]$.

Whilst it is widely accepted that non-critical medical equipment items are not meant to be sterile, the impact of frequent-touch personal and medical equipment items in the transmission of infectious disease in clinical settings should be actively managed (NHMRC). In this study, students enrolled in undergraduate courses where infection control is paramount to patient health were asked to self-report their own practises, as well as swab a piece of their own equipment, to determine its cleanliness. Our results suggest that certain medical and personal equipment items are more likely to harbour microorganisms than others. Generally, items worn on the person's body (e.g. clothes and corrective eyewear) or carried around and handled regularly throughout the day (e.g. mobile phones) were most likely to be contaminated with viable microbes, compared to items that are used irregularly or only for short periods of time in a medical context (e.g. pens, pen lights, or scissors), which can be considered to be the "cleanest". This observation is consistent with previous reports [5]. Although we hypothesised that students who cleaned their items more regularly, or believed that following infection control guidelines was important, would have a reduced microbial load and diversity recovered from their personal medical equipment, our data does not support this. It is possible that belief does not always translate into action, and that for students who do actively clean their equipment, recontamination occurs rapidly. Combined with our data and the knowledge that re-contamination of equipment after disinfection can occur and is correlated with hand hygiene behaviour in the clinical setting we plan to make more explicit reference to the concept of crosscontamination in future practical activities [9].

Infection control practices can be improved in trainee HCWs. A limitation of this project was that we did not survey our students at the completion of the activity to assess the impact of visualising the microbiological load on personal and medical equipment items pre- and postdisinfection using a standardised survey tool. To fully evaluate this strategy as a learning activity, this aspect should be included in future research. In the absence of a post-activity survey, we analysed anecdotal evidence provided as anonymous feedback on unit learning activities, which highlighted the value and impact of the practical activity for improving infection prevention and control training in undergraduate HCW students (Supplementary Table 3 ). The potential benefits of delivering infection control activities where the students and their personal or medical equipment items are the subject, increases awareness and opens an avenue for discussing infection control compliance in a low-risk environment. Appropriate infection control practices that prevent the transmission of infectious organisms are critical in reducing the incidence of HAIs. These measures are the shared responsibility of every individual working in or visiting a healthcare facility, including trainee HCWs. The number of preventable HAIs has been reduced as a result of improved campaigns targeting $\mathrm{HCW}$ compliance with infection control procedures such as hand hygiene $[4,17]$. The data generated by this study has the potential to impact industry practice and improving compliance through increased awareness of deficits in student $\mathrm{HCW}$ hygiene behaviours related to personal medical equipment.

\section{Conclusions}

Student perceptions of infection control procedures do not always correlate with infection control practice. This project utilised an inquiry-based approach to enable clinical sciences students to analyse their own personal and medical equipment items as potential vectors for the transmission of infectious diseases. The data generated by this project created an impetus for focus on infection prevention and control as part of quality care, and in our cohort translated to improved risk assessment of infectious disease transmission opportunities and understanding of the invisible world of microbiology, and increased compliance with infection prevention and control 
strategies such as hand hygiene, and PPE selection and correct donning/doffing procedures over the duration of the unit. Infection control education of undergraduate healthcare students requires the inclusion of practical microbiology activities to ensure successful translation into clinical practice.

\section{Abbreviations}

CFU: Colony-forming unit; HAl: Healthcare-associated infection; HCW: Healthcare worker; TNTC: Too numerous to count.

\section{Supplementary Information}

The online version contains supplementary material available at https://doi. org/10.1186/s12909-021-03048-1.

\section{Additional file 1.}

Additional file 2.

\section{Authors' contributions}

EP: conceived and designed the project, contributed to the analysis and interpretation of the data, and drafted significant parts of the work. ZS: performed the statistical analysis and interpretation of the data, and drafted significant parts of the work. HP: contributed to the analysis and interpretation of the data, and contributed to writing the manuscript. JOC: contributed to the analysis and interpretation of the data, and contributed to writing the manuscript. EB: contributed to the analysis and interpretation of the data, and contributed to writing the manuscript. LW: contributed to the analysis and interpretation of the data, and contributed to writing the manuscript. JC: conceived and designed the project, contributed to the analysis and interpretation of the data, and drafted significant parts of the work. The author(s) read and approved the final manuscript.

\section{Funding}

This research did not receive any specific grant from agencies in the public, commercial, or not-for-profit sectors.

\section{Availability of data and materials}

The datasets used and/or analysed during this current study are available from the corresponding author on reasonable request.

\section{Declarations}

\section{Ethics approval and consent to participate}

Ethical approval was obtained from the Queensland University of Technology Human Research Ethics Committee, approval number 1800000074 . The return of the completed project survey was accepted as an indication of informed consent to participate in this research project. Samples were de-identified by the participants before entering samples into the study.

\section{Consent for publication}

Not applicable.

\section{Competing interests}

The authors declare that they have no competing interests.

\section{Author details}

'Queensland University of Technology, School of Biomedical Sciences, Faculty of Health, 2 George Street, Brisbane City, QLD 4000, Australia. ${ }^{2}$ Queensland University of Technology, PO Box 2434, Brisbane, Queensland 4001, Australia.

\section{References}

1. ACSQHC. Australian Commission on Safety and Quality in Health Care. Annual report 2017-18. 2018.

2. Barr N, Holmes M, Roiko A, Dunn P, Lord B. Self-reported behaviors and perceptions of Australian paramedics in relation to hand hygiene and gloving practices in paramedic-led health care. Am J Infect Control. 2017;45(7):771-8. https://doi.org/10.1016/j.ajic.2017.02.020.

3. Barr N, Holmes M, Roiko A, Dunn P, Lord B. Challenges for environmental hygiene practices in Australian paramedic-led health care: a brief report. Am J Infect Control. 2018;46(6):723-5. https://doi.org/10.1016/j.ajic.2017. 11.007.

4. Grayson ML, Stewardson AJ, Russo PL, Ryan KE, Olsen KL, Havers SM, et al. Effects of the Australian National Hand Hygiene Initiative after 8 years on infection control practices, health-care worker education, and clinical outcomes: a longitudinal study. Lancet Infect Dis. 2018;18(11):1269-77. https://doi.org/10.1016/s1473-3099(18)30491-2.

5. Haun N, Hooper-Lane C, Safdar N. Healthcare personnel attire and devices as fomites: a systematic review. Infect Control Hosp Epidemiol. 2016;37(11):1367-73. https://doi.org/10.1017/ice.2016.192.

6. Havill NL. Best practices in disinfection of noncritical surfaces in the health care setting: creating a bundle for success. Am J Infect Control. 2013:41(5 Suppl):S26-30. https://doi.org/10.1016/.jajic.2012.10.028.

7. Kramer A, Schwebke I, Kampf G. How long do nosocomial pathogens persist on inanimate surfaces? A systematic review. BMC Infect Dis. 2006;6:130. https://doi.org/10.1186/1471-2334-6-130.

8. Lecat P, Cropp E, McCord G, Haller NA. Ethanol-based cleanser versus isopropyl alcohol to decontaminate stethoscopes. Am J Infect Control. 2009;37(3):241-3. https://doi.org/10.1016/j.ajic.2008.08.006.

9. Longtin Y, Schneider A, Tschopp C, Renzi G, Gayet-Ageron A, Schrenzel J, et al. Contamination of stethoscopes and physicians' hands after a physical examination. Mayo Clin Proc. 2014;89(3):291-9. https://doi.org/10. 1016/j.mayocp.2013.11.016

10. Mitchell A, Dealwis N, Collins J, Chew K, Taylor R, Schwab U, et al. Stethoscope or 'Staphoscope'? Infection by auscultation. J Hosp Infect. 2010;76(3):278-9. https://doi.org/10.1016/j.jhin.2010.06.015.

11. NHMRC. Australian guidelines for the prevention and control of infection in healthcare. Commonwealth of Australia. 2010.

12. O'Flaherty N, Fenelon L. The stethoscope and healthcare-associated infection: a snake in the grass or innocent bystander? J Hosp Infect. 2015;91(1):1-7. https://doi.org/10.1016/j.jhin.2015.04.010.

13. O'Meara P, Ruest M, Stirling C. Community paramedicine: higher education as an enabling factor. Australas J Paramed. 2014;11(2). Available from: https://ajp.paramedics.org/index.php/ajp/article/view/22

14. Storr J, Twyman A, Zingg W, Damani N, Kilpatrick C, Reilly J, et al. Core components for effective infection prevention and control programmes: new WHO evidence-based recommendations. Antimicrob Resist Infect Control. 2017;6:6. https://doi.org/10.1186/s13756-016-0149-9.

15. Team, R. C.. R: a language and environment for statistical computing. R Foundation for Statistical Computing. 2017. Retrieved from http:// www.R-project.org/. http://www.R-project.org/.

16. Uneke CJ, Ogbonna A, Oyibo PG, Ekuma U. Bacteriological assessment of stethoscopes used by medical students in Nigeria: implications for nosocomial infection control. World Health Popul. 2008;10(4):53-61 Retrieved from.

17. WHO. WHO guidelines on hand hygiene in health care. 2009. Retrieved from.

\section{Publisher's Note}

Springer Nature remains neutral with regard to jurisdictional claims in published maps and institutional affiliations. 